\title{
Heart Rate Response to Transient Chemoreceptor Stimulation in Term Infants Is Modified by Exposure to Maternal Smoking
}

\author{
SIGNE SØVIK, KRISTIN LOSSIUS, AND LARS WALLØE \\ Institute of Physiology, University of Oslo, NO-0317 Oslo, Norway [S.S., L.W.]; and Section of \\ Neonatology, Department of Paediatrics, Trondheim University Hospital, NO-7005 Trondheim, Norway \\ [K.L.].
}

\begin{abstract}
Modulation of heart rate (HR) during transient hyperoxia, hypoxia, and hypercapnia was studied in 46 healthy term infants on 103 occasions (postnatal d 2 to 82). Twenty-three infants had smoking mothers (median, 11 cigarettes/d). Transient chemoreceptor stimuli $\left(100 \% \mathrm{O}_{2}, 15 \% \mathrm{O}_{2}\right.$, or $\left.3 \% \mathrm{CO}_{2}\right)$ were presented repeatedly during quiet sleep. Beat-by-beat HR and breath-bybreath ventilation were recorded continuously. The coherently averaged HR and ventilation responses to each stimulus were calculated for each infant at each age. Outcome variables (HR change from baseline to end of stimulation, maximum HR change, and time to half-maximum) were analyzed by ANOVA. Overall, HR declined during hyperoxia (median change, 4.2 beats/min) and rose during hypoxia (median change, 4.2 beats/ min) and hypercapnia (median change, 4.6 beats/min). The percentage change in HR was positively correlated with the percentage change in ventilation $(p<0.001)$. Increasing number of cigarettes smoked by the mother was correlated with deeper HR declines and smaller HR rises $(p=0.02)$. For the population as
\end{abstract}

\section{ABSTRACT}

a whole, the HR response lagged $3.8 \mathrm{~s}$ behind the ventilatory response during hyperoxia and hypoxia $(p<0.001)$, whereas during hypercapnia there was no significant lag. The lag in HR response in the smoke-exposed group was $2.5 \mathrm{~s}$ greater than that in the control group for all three stimuli $(p=0.001)$, and the difference increased with the number of cigarettes smoked by the mother $(p<0.01)$. Both pulmonary reflexes and the type of the chemoreceptor stimulus seemed to influence HR. Maternal smoking affected the magnitude and time-course of the HR response in a dose-dependent manner. (Pediatr Res 49: 558-565, 2001)

HR, heart rate

Abbreviations:

fR, respiratory rate

$\mathbf{V}_{\mathrm{T}}$, tidal volume

$\dot{\mathbf{V}}_{\mathbf{E}}$, ventilation
In fetal life, peripheral chemoreceptor stimulation by hypoxia or hypercapnia produces bradycardia and peripheral vasoconstriction $(1,2)$. After birth, however, the same stimuli cause hyperventilation, tachycardia, and peripheral vasodilation (3-5). These responses are thought to result from an interaction of reflexes. The primary cardiac effect of chemoreceptor stimulation is bradycardia, mediated via the vagal innervation of the heart. However, a concomitant increase in $\dot{V}_{E}$ will increase lung stretch receptor activity, give slight hypocapnia, and affect central respiratory neurons, and these responses together cause an overriding tachycardia. This inter-

Received April 25, 2000; accepted October 26, 2000.

Correspondence and reprint requests: Signe Søvik, Department of Physiology, Institute of Basic Medical Sciences, University of Oslo, P.O. Box 1103 Blindern, NO-0317 Oslo, Norway; e-mail: signe.sovik@basalmed.uio.no

Supported by The Norwegian Research Council, the Norwegian Academy of Science and Letters, the Norwegian SIDS Foundation and the Norwegian National Health Association. action has been described in a number of species, including primates $(4-8)$.

In anesthetized animals, elaborate experiments can be performed in which marked changes in arterial $\mathrm{O}_{2}$ and $\mathrm{CO}_{2}$ content are introduced or the peripheral chemoreceptors are stimulated chemically or electrically. Cardiovascular effects may then be assessed while $\dot{V}_{\mathrm{E}}$ is kept constant, the degree of lung inflation is controlled, or pulmonary afferent nerves are cut or pharmacologically blocked. It is not possible to achieve a similar level of control in noninvasive studies in humans. However, trained subjects can voluntarily keep their $\dot{V}_{\mathrm{E}}$ constant, and in some pathologic conditions breathing movements are temporarily absent. Experiments making use of these phenomena have given more indirect indications of an interaction between the effects of chemoreceptor activity and lung inflation on HR in healthy adults (9), in adult ventilator-requiring quadriplegics $(10,11)$, and in preterm infants during apnea (12). In healthy uncooperating subjects, the different physiologic effects are more difficult to isolate. The effect on HR of 
chemoreceptor input and lung inflation in infants has mainly been studied during long-term changes in inspiratory gas, approaching steady-state conditions (13-17).

Our first hypothesis in this study was that infant HR modulation during a transient ventilatory chemoresponse differs according to whether the response was elicited by changes in the fraction of inspired $\mathrm{O}_{2}$ or by changes in $\mathrm{CO}_{2}$. The timing of experiments was selected to enable detection of any maturation of responses during the first few weeks of life. Peripheral chemoreceptors undergo a marked postnatal resetting of oxygen sensitivity (18), but little is known about whether this rapid development affects the cardiovascular branch of the chemoreceptor reflex or its interplay with other reflexes.

Our second hypothesis was that the magnitude or timecourse of infant HR responses to transient chemoreceptor stimuli are modified by maternal smoking during pregnancy. Nicotine has been shown to affect both peripheral chemoreceptor and central nervous transmitter level and turnover (1921). Prenatal nicotine exposure impaired the cardiac response to hypoxia in newborn rats (22), and habitual smokers displayed altered cardiorespiratory reflexes (23-26).

Because this study looked at healthy term infants during normal sleep, it was clearly not possible to standardize basal HR, basal breathing pattern, and the magnitude of the ventilatory response to the chemoreceptor stimulus. We therefore attempted to use statistical methods to separate the effect of different inputs on the integrated HR response.

\section{METHODS}

Subjects. Twenty-three randomly selected infants of women who reported having smoked $\geq 1$ cigarette per day throughout pregnancy were recruited, together with 23 control infants born to nonsmokers. Inclusion criteria were healthy infant, birth weight $\geq 2500 \mathrm{~g}$, Apgar scores $\geq 8$ at 1 and $5 \mathrm{~min}$, spontaneous vaginal delivery to term, and uncomplicated pregnancy. Exclusion criteria were any chronic maternal illness, receptoractive medication during pregnancy, and alcohol or illicit drug abuse. Eligibility for and use of prenatal health care were uniform in the unselected population studied. Regional Ethics Committee approval and written informed consent from parents were obtained, and mothers were often present during experiments.

The 46 infants were divided into two study groups. Infant and maternal characteristics are listed in Table 1. Smokeexposed and control infants did not differ with respect to maternal age, gestational age, birth weight, or postnatal age at the times of study. The two study groups received different chemoreceptor stimuli and were investigated at different postnatal ages (Table 2). Our findings on the development of ventilatory responses to changing oxygen level in group 1 has been published previously (27). Four women, all nonsmokers, declined to participate.

Mothers were asked to estimate the daily number of cigarettes smoked during pregnancy (Table 1). The reported daily number of cigarettes smoked was always equal to or higher than the figure in the medical record, which was given at the first prenatal checkup at approximately $8-10 \mathrm{wk}$ of gestation. When asked to grade their exposure to passive smoking during pregnancy, 18 of 23 nonsmoking women reported no exposure, three infrequent exposure, two some degree of exposure, and zero reported heavy exposure. In contrast, eight of 23 smoking women reported heavy exposure to passive smoking (in addition to smoke from their own cigarettes). 22 of 23 smokers continued smoking throughout the study, and 20 of 23 smokers continued breast-feeding (as did all nonsmokers). Infant hair nicotine measurements were available for study group 1 (27); measurements at $10 \mathrm{wk}$ of age showed no overlap between the exposure and the control groups, and good correlation with maternal reports of smoking $\left(r^{2}=0.63\right)$. We put no restrictions on smoking before the experiments.

Procedures. Full details of this method of assessing chemoreceptor reflexes in infants have been published previously (28). The infants were studied during a daytime nap in a cot. Three- and 10-d-old infants in study group 1 lay in the lateral position; the others slept supine. Inspiratory gases were supplied through a light-weight, handheld face mask throughout the experiment. Gases were delivered dry at room temperature; the flow was $4.65 \mathrm{~L} / \mathrm{min}$ for group 1 and $6.25 \mathrm{~L} / \mathrm{min}$ for group 2. Computer-controlled electromagnetic valves allowed instantaneous switching between breathing gas mixtures of different composition. During a full sleep period, repeated chemoreceptor challenges were presented (Table 2). The sequence of stimuli was block randomized if two kinds of stimulus were used in the same experiment. Response to hypercapnia was tested with $3 \% \mathrm{CO}_{2} / 21 \% \mathrm{O}_{2} / 76 \% \mathrm{~N}_{2}$ for $15 \mathrm{~s}$. Response to hyperoxia was tested with $100 \% \mathrm{O}_{2}$ for $15 \mathrm{~s}$. Response to hypoxia was tested with $15 \% \mathrm{O}_{2} / 85 \% \mathrm{~N}_{2}$ for $15 \mathrm{~s}$ in group 1 . However, because this stimulus proved somewhat weak (27), 20 -s periods of $15 \% \mathrm{O}_{2} / 85 \% \mathrm{~N}_{2}$ and a higher bias flow was used in group 2.

Table 1. Infant and maternal characteristics

\begin{tabular}{|c|c|c|c|c|}
\hline \multirow[b]{2}{*}{ Characteristic } & \multicolumn{2}{|c|}{ Group 1: hyperoxia and hypoxia } & \multicolumn{2}{|c|}{ Group 2: hypercapnia and hypoxia } \\
\hline & Smoke-exposed & Controls & Smoke-exposed & Controls \\
\hline Males:females & $9: 8$ & $6: 9$ & $2: 4$ & $6: 2$ \\
\hline Birth weight (g) & $3650(3150-4340)$ & $3610(2700-4220)$ & $3764(2890-4240)$ & $3879(3620-4918)$ \\
\hline Gestational age (wk:d) & $40: 3(38: 2-41: 4)$ & $39: 6(38: 1-42: 2)$ & $40: 2(38: 1-40: 8)$ & $39: 5(39: 2-41: 3)$ \\
\hline Maternal age (y) & $28.0(24-40)$ & $30.0(24-41)$ & $31.5(24-34)$ & $30.4(27-35)$ \\
\hline Maternal schooling $(\mathrm{y})^{*}$ & $12(9-18)$ & $15.5(14-17)$ & $12(11-12)$ & $15(15-17)$ \\
\hline Cigarettes/d & $10(2-22)$ & 0 & $13(1-20)$ & 0 \\
\hline
\end{tabular}

Values are medians with ranges.

$* p<0.01$ by Mann-Whitney $U$ test. 
Table 2. Experimental protocol and baseline HR values

\begin{tabular}{ccccccc}
\hline \multirow{2}{*}{$\begin{array}{c}\text { Study } \\
\text { group }\end{array}$} & $\begin{array}{c}\text { Age at } \\
\text { examination }\end{array}$ & Stimulus & $\begin{array}{c}\text { Duration (s) } \\
\text { (stimulus:air) }\end{array}$ & $\begin{array}{c}\text { Successful runs } \\
\text { (exposed:controls) }\end{array}$ & \multicolumn{2}{c}{ HR during air breathing (beats/min)* } \\
\cline { 6 - 7 } & $49 \mathrm{~h}(40-76)$ & $3 \% \mathrm{CO}_{2}$ & $15: 80$ & $6: 8$ & $106.4(89.3-115.9)$ & $110.0(102.9-119.4)$ \\
1 & $70 \mathrm{~h}(59-101)$ & $100 \% \mathrm{O}_{2}$ & $15: 60$ & $17: 12$ & $114.2(82.5-137.5)$ & $114.8(84.6-128.4)$ \\
1 & $10 \mathrm{~d}(9-12)$ & $100 \% \mathrm{O}_{2}$ & $15: 60$ & $16: 11$ & $142.2(104.0-165.4)$ & $141.7(105.3-158.3)$ \\
2 & $33 \mathrm{~d}(27-41)$ & $3 \% \mathrm{CO}_{2}$ & $15: 80$ & $5: 4$ & $130.1(116.8-151.9)$ & $134.5(123.9-141.5)$ \\
& & $15 \% \mathrm{O}_{2}$ & $20: 80$ & $5: 5$ & & \\
1 & $71 \mathrm{~d}(64-82)$ & $100 \% \mathrm{O}_{2}$ & $15: 60$ & $15: 8$ & $126.7(105.2-141.7)$ & $128.1(118.3-146.7)$ \\
\end{tabular}

Values are medians with ranges.

* Baseline heart rate differed among ages (ANOVA, $p<0.001$ ), but not between exposure groups.

Beat-by-beat HR was determined from three-lead ECG. $\dot{\mathrm{V}} \mathrm{E}$, $V_{T}$, and $f_{R}$ were calculated for each breath cycle from a pneumotachograph connected to the mask (28). For group 1, the signal of a strain-gauge band placed immediately above the umbilicus was used to calculate $f_{R}, V_{T}$ was found as the peak-trough amplitude of the signal, and $\dot{V}_{\mathrm{E}}$ was expressed in arbitrary units per minute $\left[f_{R} \times V_{T}\right]$ (27). For group 2, continuous measurements of $\mathrm{CO}_{2}$ and $\mathrm{O}_{2}$ in front of the nostrils were obtained by infrared spectroscopy and paramagnetic method, respectively (Artema Multigas Monitor MM201, Stockholm, Sweden; sampling rate $150 \mathrm{~mL} / \mathrm{min}$ ). Timed gas valve status was stored together with the physiologic data.

Data selection. Epochs of quiet sleep were selected on the basis of behavioral criteria (29). We discarded chemoreceptor stimulation periods in which a sigh disturbed the respiratory pattern for more than half of the $20 \mathrm{~s}$ immediately preceding stimulation or occurred within $30 \mathrm{~s}$ of the onset of stimulation. A sigh was defined as a breath cycle with a markedly higher peak-trough amplitude than the average breath in that epoch, followed by $>5 \mathrm{~s}$ of apnea or extremely shallow breathing movements. Smaller sighs and exaggerated breaths were common components of the ventilatory responses to hypoxia and especially to hypercapnia, and did not result in exclusion of these responses from the results. Responses in which pneumotachograph recordings indicated that mask leakage had occurred were discarded. Gas analysis measurements were inspected to ensure that the inspired concentration of $\mathrm{O}_{2}$ during hypoxic stimulation did not exceed $15 \%$ and that the inspired concentration of $\mathrm{CO}_{2}$ during hypercapnic stimulation never fell below $3 \%$. This would have happened if peak inspiratory flow had exceeded the flow of delivered breathing gas, resulting in dilution of the stimulus with room air. There was no such dilution during any accepted quiet sleep response.

Calculations. The beat-by-beat HR recordings were inspected, and artifacts due to T-wave triggering or single missed $\mathrm{R}$ waves were removed manually by interpolation. HR and ventilatory data were converted to $5-\mathrm{Hz}$ time series before further calculations. The $5-\mathrm{Hz}$ recordings in a time window from $50 \mathrm{~s}$ before to $150 \mathrm{~s}$ after the onset of each accepted stimulation period were aligned so that the onset of stimulus coincided, and were averaged (30) (Fig. 1). Coherently averaged $H R, \dot{V}_{E}, V_{T}$, and $f_{R}$ responses were calculated for each infant, age, and kind of stimulus.

Averaged responses were analyzed in a virtual instrument programmed in LabVIEW 5.0 (National Instruments Corp.,
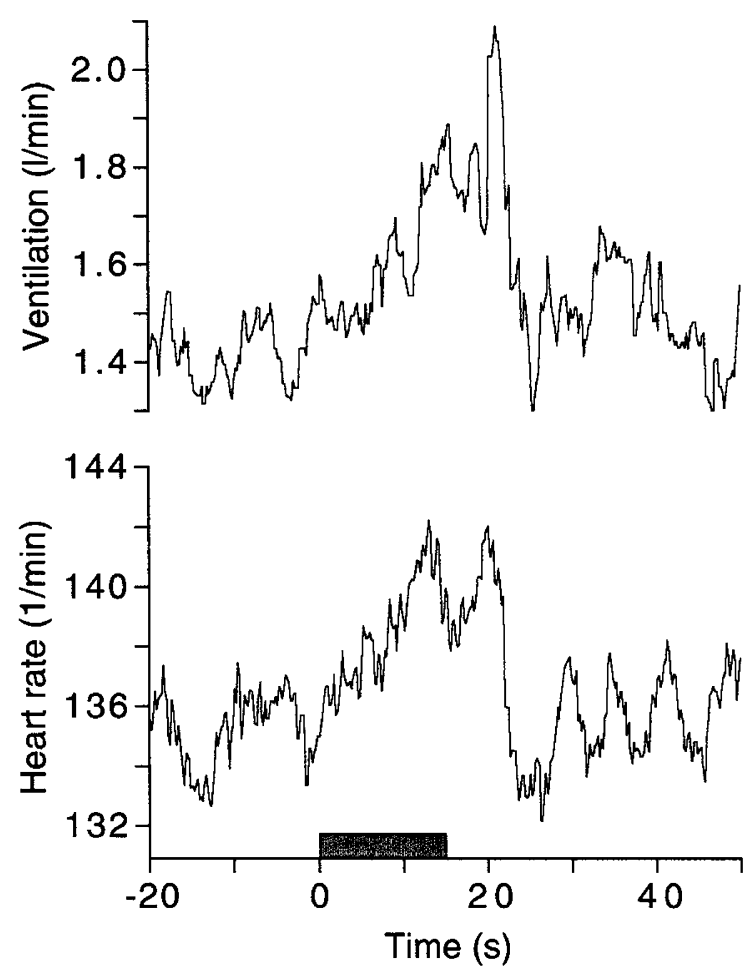

Figure 1. Closely coupled modulation of heart rate and $\dot{V}_{E}$ response to $3 \%$ $\mathrm{CO}_{2}$ (box) in a 2-d-old infant. Curves are coherent averages of six consecutive responses. Spontaneous oscillations in $\mathrm{HR}$ and $\dot{\mathrm{V}}_{\mathrm{E}}$ were typically present, superimposed on the response to chemoreceptor stimuli.

Austin, TX, U.S.A.). The median value in the $20 \mathrm{~s}$ preceding stimulation was taken as the baseline value. As a robust measure of response magnitude, we used the median value in the period $\pm 5 \mathrm{~s}$ from the end of stimulation, normalized with respect to the baseline value. The time course of $H R$ and $\dot{V}_{E}$ responses was assessed after removal of high-frequency variation with a 20-order low-pass Butterworth filter with a cutoff frequency of $0.20 \mathrm{~Hz}$. Time distortion of the signal was avoided by running the data forward and then backward again through the filter. The extreme HR and $\dot{V}_{E}$ values and the time at which they occurred in the resulting filtered responses were determined automatically, the search being limited to the first $30 \mathrm{~s}$ after the start of stimulation. The value halfway between the baseline and the extreme value was computed, and its time of occurrence on the filtered curve was found. The virtual instrument was set to detect low extremes in data from hyperoxic stimulation and high extremes in data from hypoxic or 
hypercapnic stimulation. Plots of the unfiltered data and the filtered version with calculated time points indicated were always inspected to ensure a sensible fit. The extreme values of HR and $\dot{V}_{E}$ were normalized with respect to the baseline value. The timing of responses was determined as the time from start of stimulation to the half-extreme point. This point occurred on a steep part of the curve, making it a more robust measure than the time of extreme response. The difference in timing between HR and $\dot{V}_{E}$ responses was expressed as the time to halfextreme $_{\mathrm{HR}}$ minus the time to half-extreme $\dot{\mathrm{V}}_{\mathrm{E}}$.

Statistical analysis. Values are medians and ranges unless otherwise stated. For multiway analysis, we used a general mixed model of variance (BMDP 3V, release 7.0, 1993, BMDP Statistical Software Inc., Cork, Ireland), which allows repeated-measures ANOVA to be applied to unbalanced data sets with missing observations. We analyzed how the maximum HR response during transient chemoreceptor stimulation was affected by stimulus type; baseline HR and $\mathrm{fR}_{\mathrm{R}}$; maximum $\dot{\mathrm{V}}_{\mathrm{E}}$, $V_{T}$, and $f_{R}$ response; number of cigarettes smoked per day by the mother; and age group. Median HR at the end of stimulation and the delay in appearance of the HR response relative to the $\dot{V}_{\mathrm{E}}$ response were analyzed for effects of the same independent variables. Age group was considered a categorical variable; the other independent variables were modeled as linear covariates. Analyses were also run with smoke exposure as a categorical (yes/no) variable.

\section{RESULTS}

On 103 occasions we obtained at least one accepted stimulation period during quiet sleep (Table 2). The median number of stimulus repetitions was 6 (range, 1-13) for hyperoxia, 6 (range, 1-11) for hypoxia, and 6 (range, 1-10) for hypercapnia. Baseline HR differed significantly among postnatal ages $(p<$ 0.001 , Table 2) but was unaffected by smoke exposure. There was good correlation between the two variables used to estimate the magnitude of the HR response, i.e. the difference between the baseline and maximum response values and between the baseline value and the value at the end of stimulation $\left(r^{2}=0.88\right)$. Experiments started $63 \mathrm{~min}$ (range, 15-187 $\left.\mathrm{min}\right)$ after the start of the preceding meal, similar for the two exposure groups. Proximity to a meal did not affect the measured variables.

Magnitude of HR changes during transient chemoreceptor stimulation. HR declined during hyperoxia and rose during hypoxia and hypercapnia. The maximum change in HR was positively correlated with the maximum change in $\dot{V}_{\mathrm{E}}(p<$ 0.001 , Fig. 2). Compared with the control group, the smokeexposed group tended to show larger HR declines when $\dot{V}_{E}$ decreased (hyperoxia) and smaller HR rises when $\dot{V}_{E}$ increased (hypoxia and hypercapnia) ( $p=0.05$, Figs. 2 and 3$)$. When the number of cigarettes smoked by the mother was used as explanatory variable in the regression, the effect of smoking on maximum change in HR during chemoreceptor stimuli was found to be significant $(p=0.022)$.

The above results were confirmed in the analysis of median HR at the end of stimulation (Table 3). This more robust measure of the HR response was positively correlated with the

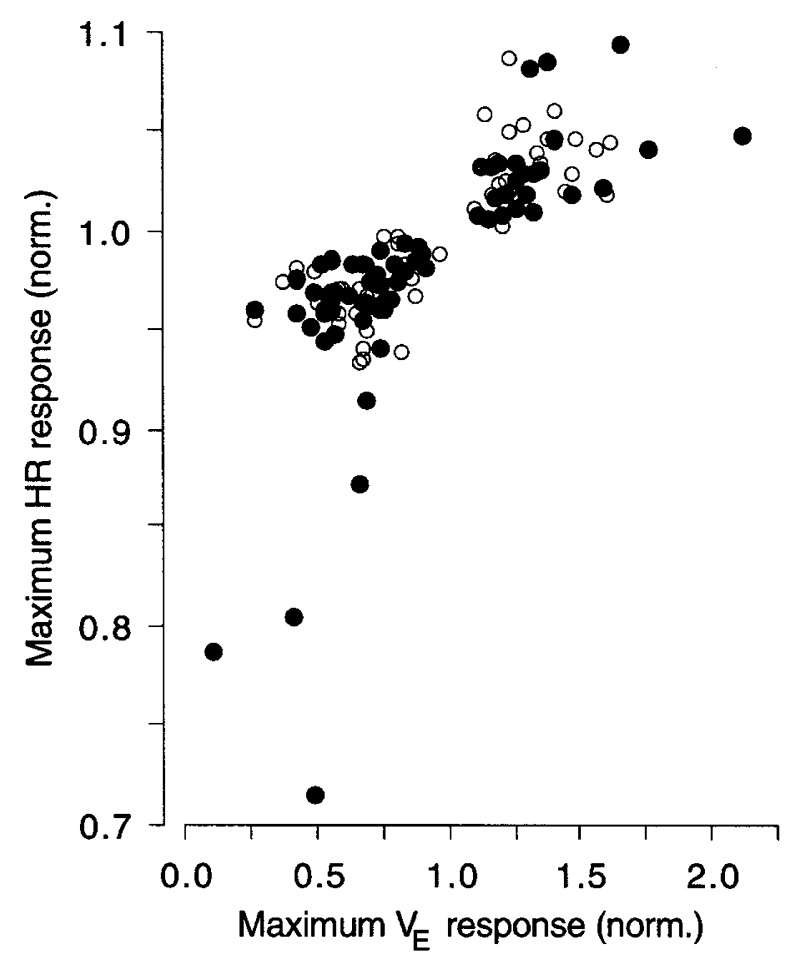

Figure 2. Relationship between maximum HR response and maximum $\dot{V}_{E}$ response to transient changes in breathing gas. Stimulation with $100 \% \mathrm{O}_{2}$ (maximum $\mathrm{HR}$ response, $<1$ ), $15 \% \mathrm{O}_{2}$, or $3 \% \mathrm{CO}_{2}$ (maximum $\mathrm{HR}$ response $>1$ ). Data are from 46 infants aged 2-82 d, studied on 103 occasions. Filled circles indicate infants of smoking mothers. $\mathrm{HR}_{\text {extreme }}=0.92+(0.09 \times$ $\dot{\mathrm{V}}_{\mathrm{E}}$ extreme), $r^{2}=0.70$ (the four infants with deepest HR declines were excluded from the regression).

magnitude of the ventilatory response $(p<0.001)$ and negatively correlated with the number of cigarettes smoked by the mother $(p=0.038)$. To ensure that the observed effect of exposure to cigarette smoke did not result solely from a few extreme responses, the material was divided in quartiles according to the magnitude of the HR decline during hyperoxia. The median number of cigarettes per day smoked by the mother increased from the first to the fourth quartile $(10 / \mathrm{d}$, $10 / \mathrm{d}, 12 / \mathrm{d}$, and 15/d). Moreover, the proportion of smokers reporting heavy exposure to passive smoking was largest in the fourth quartile $(0.42$ as compared with $0.33,0.29$, and 0.27 in the first through third quartiles).

Stimulus type and postnatal age did not add any significant effect on the magnitude of the maximum HR response, probably because it depended so closely on the stimulus- and age-related VE response. Hyperoxia lowered HR by 3.7 beats/ $\mathrm{min}, 4.3$ beats $/ \mathrm{min}$, and 4.8 beats $/ \mathrm{min}$ at the three ages studied, paralleling the development of ventilatory responses (27). Hypoxia for $15 \mathrm{~s}$ (wk 10) raised HR by 2.6 beats/min, whereas hypoxia for $20 \mathrm{~s}$ (wk 4) raised HR by 4.2 beats/min. Hypercapnia raised HR by 4.6 beats/min on $\mathrm{d} 2$ and by 3.8 beats/min in wk 4. For hyperoxia and hypoxia, the smoke-exposed group's median change in HR was smaller than that of the control group at all ages tested (deeper HR declines and smaller HR rises). For hypercapnia, the smoke-exposed group's median HR rise was smaller than that of the control group at wk 4, but not at postnatal d 2 . 


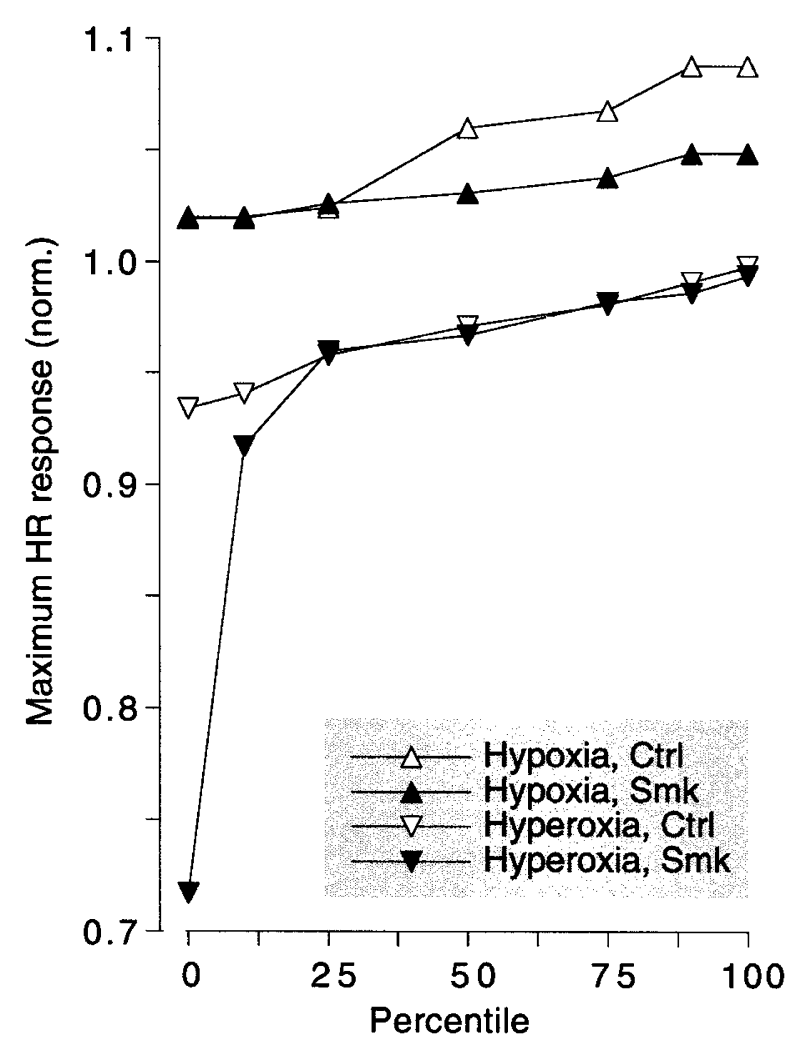

Figure 3. Cigarette smoke exposure affected the magnitude of HR responses. Distribution of normalized HR responses to 20-s hypoxia (upper two curves) and 15-s hyperoxia (lower two curves) in infants exposed to maternal smoking (Smk, black triangles) and unexposed control infants (Ctrl, white triangles). During hypoxia, HR rises were smaller in the smoke-exposed group than in control infants for all responses $>25$ th percentile. During hyperoxia, HR declines were deeper in the smoke-exposed group than in control infants for responses $<25$ th percentile.

Basal HR and $\mathrm{fR}_{\mathrm{R}}$ were also age-related, which may have been why neither affected the HR response significantly. $\dot{V}_{E}$ changes were primarily achieved by changing $\mathrm{V}_{\mathrm{T}}\left(r^{2}=0.82\right)$, not $f_{R}\left(r^{2}=0.005\right)$. Therefore, with $\dot{V}_{E}$ change included as an explanatory variable, neither $\mathrm{V}_{\mathrm{T}}$ nor $\mathrm{fR}_{\mathrm{R}}$ change contributed to the model.

Timing of HR response relative to the $\dot{V}_{E}$ response. The HR response followed the $\dot{V}_{E}$ response after a delay, and the magnitude of this time lag depended on the stimulus type. For the study as a whole, the HR decline during hyperoxia and the HR rise during hypoxia lagged $3.8 \mathrm{~s}(95 \%$ confidence interval, $3.0-4.7 \mathrm{~s}$ ) behind the $\dot{V}_{\mathrm{E}}$ response, and there was no difference between the two stimuli $(p=0.28)$. In contrast, the HR rise during hypercapnia was not delayed relative to the $\dot{V}_{\mathrm{E}}$ response, the median lag being $0.7 \mathrm{~s}(95 \%$ confidence interval, -0.9 to $2.4 \mathrm{~s}$ ). This was significantly different from the lag during both hyperoxia $(p<0.001)$ and hypoxia $(p=0.045)$.

The time lag between $\dot{V}_{\mathrm{E}}$ and HR responses was $2.5 \mathrm{~s}$ longer in the smoke-exposed group than in the control group $(p=$ 0.001). This difference was similar for all three chemoreceptor stimuli (Fig. 4) and for all postnatal ages tested. The magnitude of the time lag increased by approximately $0.2 \mathrm{~s}$ per cigarette per day smoked by the mother $(p<0.01)$. Postnatal age, basal HR and $f_{R}$, and the magnitude of $\dot{V}_{E}$ response did not affect the timing of the HR response.
Spontaneous oscillations in HR and $\dot{V}_{E .}$. In all but three runs, spontaneous oscillations in HR and $\dot{V}_{\mathrm{E}}$ were apparent in the filtered coherently averaged responses (Fig. 1). The cycle period during air breathing was measured manually and found to be $7.2 \mathrm{~s}$ (range, 5.2-13.0 s) for $\mathrm{HR}$ and $7.2 \mathrm{~s}$ (range, $4.8-12.4 \mathrm{~s}$ ) for $\dot{\mathrm{V}}_{\mathrm{E}}$, i.e. $0.077-0.208 \mathrm{~Hz}$.

\section{DISCUSSION}

This study illustrates a close interplay between cardiac and respiratory reflexes in developing infants. We found that the relative time course of $\dot{\mathrm{V}}_{\mathrm{E}}$ and $\mathrm{HR}$ responses differed according to whether the stimulus was a change in $\mathrm{O}_{2}$ or $\mathrm{CO}_{2}$. Also, our findings indicate that aspects of cardiorespiratory control may be altered in apparently healthy term infants exposed to moderate levels of maternal cigarette smoke pre- and postnatally.

Mechanisms explaining the HR response to changes in inspiratory gases. The primary HR response to chemoreceptor stimulation by hypoxia or hypercapnia is bradycardia. However, the concurrent ventilatory response increases lung stretch receptor activity, which has the opposite effect on cardiac vagal motoneurons and causes an overriding tachycardia $(4,5)$. Hyperoxia reduces chemoreceptor firing frequency and thereby lung stretch receptor activity, which together would be expected to decrease HR. This was elegantly demonstrated in healthy adults exposed to a transient hyperoxic relief from isoor hypercapnic hypoxia (9). Ventilation declined while the HR response was biphasic, with a slight increase in HR preceding a later fall in HR.

In this study, the HR changes during changing chemoreceptor input corresponded well with the lung stretch receptorrelated HR responses reported in previous work. During transient hyperoxia, hypoxia, or hypercapnia, $\dot{V}_{E}$ and HR were modulated in parallel (Fig. 1). The magnitude of the change in HR depended on the magnitude of the change in $\dot{V}_{\mathrm{E}}$, which was

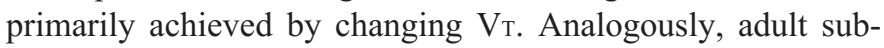
jects could eliminate or reduce the fall in HR during hyperoxia by purposely maintaining their breathing at the prestimulus level (9). In cats, stepwise increases in lung volume progressively diminished arterial chemoreceptor-induced bradycardia (6). When the magnitude of the $\dot{V}_{E}$ response was controlled for, we were unable to detect any independent effect of stimulus type (i.e. hypoxia versus hypercapnia) on the magnitude of HR response.

Relative timing of the HR and $\dot{V}_{E}$ responses. The timing of HR responses did depend on chemoreceptor stimulus type. When infants were challenged with hyperoxia or hypoxia, the ensuing HR response was delayed by $3.8 \mathrm{~s}$ relative to the $\dot{\mathrm{V}}_{\mathrm{E}}$ response. Because of the computational methods used, this may even have been a slight underestimation of the true difference in timing between efferent nerve signals. Values of instantaneous $\dot{V}_{E}$ and HR were assigned to the end of each breath and heart cycle, respectively, and breath cycles were approximately three times as long as heart cycles. Interestingly, the HR response during hypercapnia was not delayed relative to the $\dot{\mathrm{V}}_{\mathrm{E}}$ response.

Various mechanisms may underlie the delay between the $\dot{V}_{\mathrm{E}}$ and the HR response. First, the delay may have corresponded 
Table 3. Change in median HR during transient chemoreceptor stimuli

\begin{tabular}{lcc}
\hline & \multicolumn{1}{c}{ HR change from baseline to end of stimulation (beats/min) } \\
\cline { 2 - 3 } Stimulus & Smoke-exposed & Controls \\
\hline Hyperoxia & $-2.19(-2.58,-1.49)$ & $-1.91(-2.47,-1.21)$ \\
Hypoxia & $2.65(2.18,4.59)$ & $6.67(0.64,7.02)$ \\
Hypercapnia & $2.31(0.66,3.58)$ & $3.12(1.39,4.04)$ \\
\hline
\end{tabular}

Values are medians and nonparametric near-95\% confidence intervals. Stimulation lasted $15 \mathrm{~s}$ (hyperoxia and hypercapnia) and 20s (hypoxia).

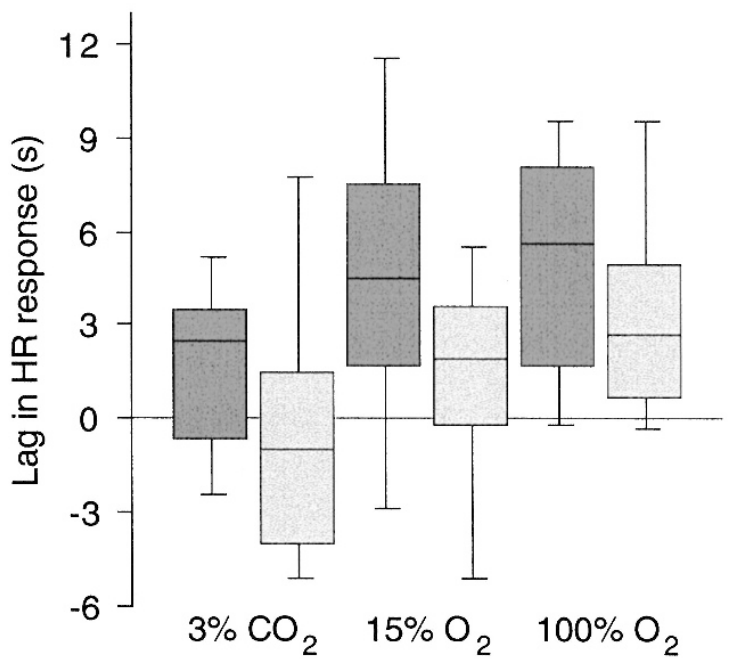

Figure 4. Cigarette smoke exposure delayed the HR responses. Time from half-maximum $\dot{V}_{E}$ response to half-maximum HR response during various chemoreceptor stimuli in smoke-exposed infants (dark boxes) and unexposed infants (light boxes). HR responses in smoke-exposed infants were delayed compared with those in control infants for all three stimuli. Overall, HR responses were significantly delayed relative to $\dot{V}_{E}$ responses during high and low oxygen but not during hypercapnia. Spontaneous oscillations in HR and $\dot{V}$ E resulted in some widely scattered values.

to a period when the opposing influences of arterial chemoreceptors and lung stretch receptors on cardiac vagal motoneurons counterbalanced each other. Differences in stimulus strength may explain why adult subjects who experienced sudden hyperoxic relief from hypoxia displayed a biphasic HR response (9), whereas infants in our study only displayed a

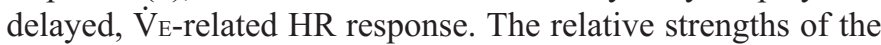
interacting reflex arcs may also differ in infants and adults. Second, central nervous processing time may have contributed to the delay. The initial HR increase associated with sighs in infants appears approximately $3 \mathrm{~s}$ after the onset of inspiration (31).

The lack of delay between the increases in $\dot{V}_{E}$ and HR when the response was elicited by $\mathrm{CO}_{2}$ requires further explanation. However, differences between carotid and aortic body chemoreceptors may be partly responsible. Aortic body chemoreceptors produce similar cardiovascular responses to carotid body chemoreceptors, but much smaller ventilatory responses (32), and are relatively insensitive to changes in $\mathrm{CO}_{2}(33,34)$. Thus, the direct chemoreceptor influence on cardiac vagal motoneurons may have been weaker during hypercapnia than during hypoxia and hyperoxia, allowing HR to rise simultaneously with $\dot{V}_{E}$.

Dose-dependent effects of cigarette smoke exposure. Baseline HR during air breathing showed clear changes with post- natal age, similar in the two exposure groups (Table 2). In contrast, the modulation of HR during transient changes in inspiratory $\mathrm{O}_{2}$ and $\mathrm{CO}_{2}$ was affected by cigarette smoke exposure in a dose-dependent manner. Analogously, neonatal rats exposed prenatally to nicotine equivalent to 20 cigarettes/d showed unaffected baseline HR but precipitous bradycardia during hypoxia (22). Various mechanisms may underlie our findings, as nicotine has numerous sites of action. Chronic prenatal nicotine exposure markedly reduced norepinephrine turnover in rat brainstem, fore-, and midbrain (21); influenced gene expression of cholinergic nerve terminal markers as well as neuronal function (35); and altered mouse brain nicotinic binding sites (36). In humans, nicotinic acetylcholine receptors in brainstem nuclei that are thought to regulate cardiorespiratory function were abundant by mid-gestation (37).

A recent electrophysiologic study of cardiac vagal motoneurons may provide a more direct explanation. Nicotine was found to increase the excitability and efficacy of synaptic transmission in cardiac vagal motoneurons by at least three different mechanisms (38). Thus, it is possible that nicotine exposure could promote the stimulating effect of chemoreceptor input on cardiac vagal motoneurons relative to the inhibiting effect of pulmonary afferent input. This could explain why smoke-exposed infants had substantially longer delays between the $\dot{V}_{E}$ and HR responses for all three stimuli (Fig. 4), and also why smoke-exposed infants showed deeper HR declines during stimuli that lowered $\dot{V}_{E}$ and slightly smaller HR rises than control infants during stimuli that raised $\dot{V}_{\mathrm{E}}$ (Fig. 3 and Table 3).

Prenatal cigarette smoke exposure probably caused much of the observed effects. The increase in HR response delay in smoke-exposed infants was present already on postnatal d 2 and 3 , its magnitude being similar to that observed later. HR response magnitude was also affected on postnatal d 3. Still, an additional effect of postnatal smoke exposure cannot be ruled out. All but one woman who had smoked during pregnancy continued smoking after delivery, and no reported nonsmoker started smoking during the study. Therefore, effects of postnatal exposure to maternal cigarette smoking could not be separated from the prenatal effects in our study.

In any given experiment, the effects of an unknown degree of short-term nicotine exposure were added to the effects of long-term exposure. This short-term exposure was difficult to quantify. Almost all smoking women claimed they never smoked in the same room as their infant. Concerning nicotine in mother's milk, proximity to the preceding meal did not affect the infants' HR responses. However, maternal smoking in the period before this meal was not registered in detail. 
The observed effects of exposure to maternal cigarette smoking on cardiorespiratory control were probably not of clinical importance in these healthy term infants. Still, the effects were dose-dependent and consistent across type of chemoreceptor stimulus and direction of ventilatory change during a 3-month age span. Our findings are in accordance with previous work indicating that cigarette smoke exposure may influence autonomic nervous function even in apparently healthy individuals, both before birth (39), in infants (40), and in adults (23-26). In a situation with heavy exposure, individual high susceptibility, a vulnerable developmental period, or the presence of disease, it is possible that cigarette smoke exposure could affect infant HR control critically.

Extreme responders in our study may have represented particular combinations of acute exposure, individual vulnerability, and developmental immaturity. The four deepest HR declines (Fig. 2) were found in three 3-d-old infants and one 10-d-old infant who showed particularly strong $\dot{V}_{E}$ responses for their age group ( $\geq 88$ th percentile). In all infants in whom HR declined markedly, hyperoxia triggered an unstable, oscillatory pattern of both $\dot{V}_{E}$ and HR (Fig. 5). One infant who responded in this manner at $3 \mathrm{~d}$ of age had extremely periodic respiration during sleep at $10 \mathrm{~d}$. However, no extreme responder showed atypically strong responses in later experiments.

Clinical situations in which our findings may be relevant are central or obstructive apneas (12) and the laryngeal reflex
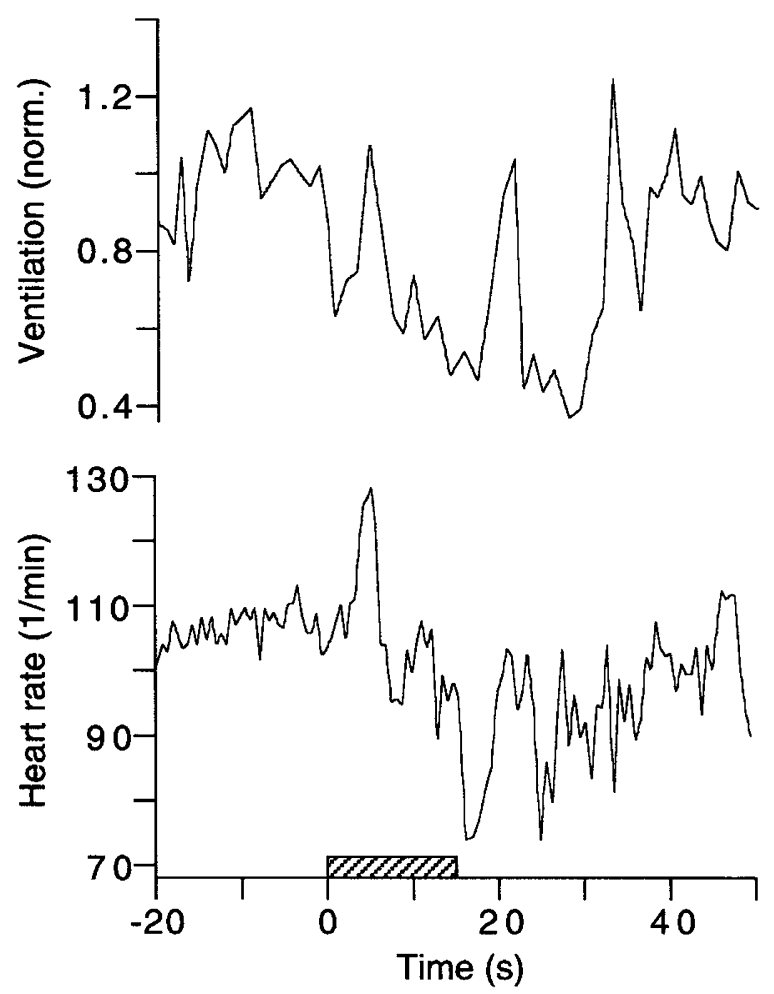

Figure 5. Exaggerated HR response to transient hyperoxia in a healthy 3-d-old boy whose mother smoked 20 cigarettes/d and father smoked 60 cigarettes/d. The abrupt fall in $\dot{V}_{E}$ at the time of gas valve clicks may have been partly an auditory response. During hyperoxia (box) VंE declined markedly; and HR declined by $>30$ beats/min and was not stabilized until $40 \mathrm{~s}$ after the stimulation ceased. response (41). In both situations, chemoreceptor input caused by rising hypoxia and hypercapnia will depress HR while stimulation of HR from the filling of lungs is absent. Whether cigarette smoke exposure could enhance this bradycardia warrants further investigation. Interestingly, prenatal cigarette smoke exposure has been shown to increase the incidence of obstructive apneas in healthy infants (42). Infants with recurrent or severe apnea frequently showed a paradoxical decrease in HR during $\mathrm{CO}_{2}$ challenge (17); however, exposure to nicotine was not reported in this study. Infant $\mathrm{HR}$ during a $\mathrm{CO}_{2}$ challenge is of interest because $\mathrm{CO}_{2}$ rebreathing during sleep has been suggested as a trigger factor in sudden infant death syndrome. Maternal smoking is the most consistent risk factor for sudden infant death syndrome.

In conclusion, we found that in apparently healthy term infants exposed to moderate levels of maternal smoking during an uncomplicated pregnancy, HR responses to transient changes in inspiratory gases were altered. We speculate that short-term nicotine effects and developmental immaturity may have enhanced the effects of long-term nicotine exposure in a few extreme responders. Further physiologic studies in healthy populations of infants are warranted.

Acknowledgment. The authors thank Torsten Eken, M.D., Ph.D., for performing the LabVIEW programming.

\section{REFERENCES}

1. Dawes GS, Duncan SL, Lewis BV, Merlet CL, Owen-Thomas JB, Reeves JT 1969 Cyanide stimulation of the systemic arterial chemoreceptors in foetal lambs. J Physiol (Lond) 201:117-128

2. Hanson M, Kumar P 1994 Chemoreceptor function in the fetus and neonate. In: O’Regan R (ed) Arterial Chemoreceptors: Cell to System. Plenum Press, New York, pp $99-108$

3. Dawes GS, Lewis BV, Milligan JE, Roach MR, Talner NS 1968 Vasomotor responses in the hind limbs of foetal and new-born lambs to asphyxia and aortic chemoreceptor stimulation. J Physiol (Lond) 195:55-81

4. Daly MB 1986 Interactions between respiration and circulation. In: Cherniack NS, Widdicombe JG (eds) Handbook of Physiology, Section 3: The Respiratory System, Vol II. American Physiological Society, Bethesda, MD, pp 529-594

5. Marshall JM 1994 Peripheral chemoreceptors and cardiovascular regulation. Physiol Rev 74:543-594

6. Daly MB, Kirkman E 1989 Differential modulation by pulmonary stretch afferents of some reflex cardioinhibitory responses in the cat. J Physiol (Lond) 417:323-341

7. Daly MB, Korner PI, Angell-James JE, Oliver JR 1978 Cardiovascular-respiratory reflex interactions between carotid bodies and upper-airways receptors in the monkey. Am J Physiol 234:H293-H299

8. Daly MB, Korner PI, Angell-James JE, Oliver JA 1978 Cardiovascular and respiratory effects of carotid body stimulation in the monkey. Clin Exp Pharmacol Physiol 5:511-524

9. Drysdale DB, Petersen ES 1977 Arterial chemoreceptors, ventilation and heart rate in man. J Physiol (Lond) 273:109-120

10. Berk JL, Levy MN 1977 Profound reflex bradycardia produced by transient hypoxia or hypercapnia in man. Eur Surg Res 9:75-84

11. Daly MB, Angell-James JE, Elsner R 1979 Role of carotid-body chemoreceptors and their reflex interactions in bradycardia and cardiac arrest. Lancet 1:764-767

12. Henderson-Smart DJ, Butcher-Puech MC, Edwards DA 1986 Incidence and mechanism of bradycardia during apnoea in preterm infants. Arch Dis Child 61:227-232

13. Brady JP, Cotton EC, Tooley WH 1964 Chemoreflexes in the newborn infant: effects of $100 \%$ oxygen on heart rate and ventilation. J Physiol (Lond) 172:332-341

14. Brady JP, Ceruti E 1966 Chemoreceptor reflexes in the newborn infant: effects of varying degrees of hypoxia on heart rate and ventilation in a warm environment. J Physiol (Lond) 184:631-645

15. Rigatto H, Brady JP, de la Torre Verduzco R 1975 Chemoreceptor reflexes in preterm infants: II. The effect of gestational and postnatal age on the ventilatory response to inhaled carbon dioxide. Pediatrics 55:614-620

16. Rigatto H, Brady JP, de la Torre Verduzco R 1975 Chemoreceptor reflexes in preterm infants: I. The effect of gestational and postnatal age on the ventilatory response to inhalation of $100 \%$ and $15 \%$ oxygen. Pediatrics 55:604-613

17. Katz-Salamon M, Milerad J 1998 The divergent ventilatory and heart rate response to moderate hypercapnia in infants with apnoea of infancy. Arch Dis Child 79:231-236

18. Blanco CE, Dawes GS, Hanson MA, McCooke HB 1984 The response to hypoxia of arterial chemoreceptors in fetal sheep and newborn lambs. J Physiol (Lond) $351: 25-37$ 
19. Holgert H, Hokfelt T, Hertzberg T, Lagercrantz H 1995 Functional and developmental studies of the peripheral arterial chemoreceptors in rat: effects of nicotine and possible relation to sudden infant death syndrome. Proc Natl Acad Sci USA 92:75757579

20. Navarro HA, Seidler FJ, Schwartz RD, Baker FE, Dobbins SS, Slotkin TA 1989 Prenatal exposure to nicotine impairs nervous system development at a dose which does not affect viability or growth. Brain Res Bull 23:187-192

21. Slotkin TA, Lappi SE, McCook EC, Lorber BA, Seidler FJ 1995 Loss of neonatal hypoxia tolerance after prenatal nicotine exposure: implications for sudden infant death syndrome. Brain Res Bull 38:69-75

22. Slotkin TA, Saleh JL, McCook EC, Seidler FJ 1997 Impaired cardiac function during postnatal hypoxia in rats exposed to nicotine prenatally: implications for perinatal morbidity and mortality, and for sudden infant death syndrome. Teratology 55:177184

23. Hayano J, Yamada M, Sakakibara Y, Fujinami T, Yokoyama K, Watanabe Y, Takata K 1990 Short- and long-term effects of cigarette smoking on heart rate variability. Am J Cardiol 65:84-88

24. Niedermaier ON, Smith ML, Beightol LA, Zukowska-Grojec Z, Goldstein DS, Eckberg DL 1993 Influence of cigarette smoking on human autonomic function. Circulation 88:562-571

25. Lucini D, Bertocchi F, Malliani A, Pagani M 1996 A controlled study of the autonomic changes produced by habitual cigarette smoking in healthy subjects. Cardiovasc Res 31:633-639

26. Gerhardt U, Vorneweg P, Riedasch M, Hohage H 1999 Acute and persistent effects of smoking on the baroreceptor function. J Auton Pharmacol 19:105-108

27. Søvik S, Lossius K, Eriksen M, Grøgaard J, Walløe L 1999 Development of oxygen sensitivity in infants of smoking and non-smoking mothers. Early Hum Dev 56:217232

28. Søvik S, Eriksen M, Lossius K, Grøgaard J, Walløe L 1999 A method of assessing ventilatory responses to chemoreceptor stimulation in infants. Acta Paediatr 88:563570

29. Prechtl HFR 1974 The behavioural states of the newborn infant: a review. Brain Res $76: 185-212$

30. Rompelman O, Ros HH 1986 Coherent averaging technique: a tutorial review. Part 1: noise reduction and the equivalent filter. J Biomed Eng 8:24-29
31. Inwald D, Hathorn MK, Costeloe K 1996 The deep breath vasoconstriction reflex: a new tool for autonomic assessment in infancy? Early Hum Dev 45:55-61

32. Daly M, Ungar A 1966 Comparison of the reflex responses elicited by stimulation of the separately perfused carotid and aortic body chemoreceptors in the dog. J Physiol (Lond) 182:379-403

33. Lahiri S, Mokashi A, Mulligan E, Nishino T 1981 Comparison of aortic and carotid chemoreceptor responses to hypercapnia and hypoxia. J Appl Physiol 51:55-61

34. Pokorski M, Mokashi A, Mulligan E, Nishino T, Lahiri S 1981 Responses of aortic chemoreceptors before and after pneumothorax in the cat. J Appl Physiol 51:665-670

35. Zahalka EA, Seidler FJ, Lappi SE, McCook EC, Yanai J, Slotkin TA 1992 Deficits in development of central cholinergic pathways caused by fetal nicotine exposure: differential effects on choline acetyltransferase activity and $\left[{ }^{3} \mathrm{H}\right]$ hemicholinium-3 binding. Neurotoxicol Teratol 14:375-382

36. van de Kamp JI, Collins AC 1994 Prenatal nicotine alters nicotinic receptor development in the mouse brain. Pharmacol Biochem Behav 47:889-900

37. Kinney HC, O'Donnell TJ, Kriger P, White WF 1993 Early developmental changes in $\left[{ }^{3} \mathrm{H}\right]$ nicotine binding in the human brainstem. Neuroscience 55:1127-1138

38. Neff RA, Humphrey J, Mihalevich M, Mendelowitz D 1998 Nicotine enhances presynaptic and postsynaptic glutamatergic neurotransmission to activate cardiac parasympathetic neurons. Circ Res 83:1241-1247

39. Sindberg Eriksen P, Gennser G, Lindvall R, Nilsson K 1984 Acute effects of maternal smoking on fetal heart beat intervals. Acta Obstet Gynecol Scand 63:385-390

40. Franco P, Chabanski S, Szliwowski H, Dramaix M, Kahn A 2000 Influence of maternal smoking on autonomic nervous system in healthy infants. Pediatr Res 47:215-220

41. Wennergren G, Hertzberg T, Milerad J, Bjure J, Lagercrantz H 1989 Hypoxia reinforces laryngeal reflex bradycardia in infants. Acta Paediatr Scand 78:11-17

42. Kahn A, Groswasser J, Sottiaux M, Kelmanson I, Rebuffat E, Franco P, Dramaix M, Wayenberg JL 1994 Prenatal exposure to cigarettes in infants with obstructive sleep apneas. Pediatrics 93:778-783 